\title{
Reduced AMP-activated protein kinase activity in mouse skeletal muscle does not exacerbate the development of insulin resistance with obesity
}

\author{
S. Beck Jørgensen • H. M. O'Neill • K. Hewitt • \\ B. E. Kemp • G. R. Steinberg
}

Received: 24 June 2009 / Accepted: 2 July 2009 /Published online: 18 August 2009

(C) Springer-Verlag 2009

\begin{abstract}
Aims/hypothesis Obesity-related insulin resistance is associated with accumulation of bioactive lipids in skeletal muscle. The AMP-activated protein kinase (AMPK) regulates lipid oxidation in muscle by inhibiting acetyl-CoA carboxylase-2 (ACC2) and increasing mitochondrial biogenesis. We investigated whether reduced levels of muscle AMPK promote lipid accumulation and insulin resistance during high-fat feeding. Methods Male C57/BL6 wild-type mice and transgenic littermates overexpressing an $\alpha 2$ AMPK kinase-dead (KD) in muscle were fed control or high-fat diet. Whole-body glucose homeostasis was assessed by glucose and insulin tolerance tests, and by measuring fasting and fed serum insulin and glucose. Insulin action in muscle was determined by measuring 2-deoxy- $\left[{ }^{3} \mathrm{H}\right]$ glucose uptake and Akt phosphorylation in incubated soleus and extensor digitorum longus muscles. Muscle triacylglycerol, diacylglycerol and ceramide content was measured by thin-layer chromatogra-
\end{abstract}

S. Beck Jørgensen and H. M. O'Neill contributed equally to this work.

S. Beck Jørgensen • H. M. O’Neill • K. Hewitt • B. E. Kemp •

G. R. Steinberg

St Vincent's Institute of Medical Research

and Department of Medicine, University of Melbourne,

Fitzroy, VIC, Australia

S. Beck Jørgensen $(\bowtie)$

Molecular Physiology Group, Section of Human Physiology,

Department of Exercise and Sport Sciences,

University of Copenhagen,

13-Universitetsparken,

2100 Copenhagen, Denmark

e-mail: sbjorgensen@ifi.ku.dk

G. R. Steinberg

Department of Medicine, McMaster University,

Hamilton, ON, Canada phy. Mitochondrial proteins were measured by immunoblotting.

Results KD mice had reduced skeletal muscle $\alpha 2 \mathrm{AMPK}$ activity $(50 \%$ in gastrocnemius and $>80 \%$ in soleus and extensor digitorum longus) and ACC2 Ser228 phosphorylation ( $90 \%$ in gastrocnemius). High-fat feeding increased body mass and adiposity, and impaired insulin and glucose tolerance; however, there were no differences between wild-type and KD littermates. High-fat feeding impaired insulin-stimulated muscle glucose uptake and Akt-phosphorylation, while increasing muscle triacylglycerol, diacylglycerol $(p=0.07)$ and ceramide, but these effects were not exacerbated in $\mathrm{KD}$ mice. In response to high-fat feeding, mitochondrial proteins were increased to similar levels in wild-type and KD muscles.

Conclusions/interpretation Obesity-induced lipid accumulation and insulin resistance were not exacerbated in AMPK KD mice, suggesting that reduced levels of muscle $\alpha 2$ AMPK do not promote insulin resistance in the early phase of obesity-related diabetes.

Keywords ACC2 - Acetyl-CoA carboxylase- 2 . 5'-AMP-activated protein kinase - AMPK - High-fat diet . HOMA-IR · Insulin resistance $\cdot$ Mouse · Obesity · Skeletal muscle

$\begin{array}{ll}\text { Abbreviations } \\ \text { ACC2 } & \text { Acetyl-CoA carboxylase-2 } \\ \text { AICAR } & \text { Aminoimidazole carboxamide ribonucleotide } \\ \text { AMPK } & \text { AMP-activated protein kinase } \\ \text { COX-1 } & \text { Cytochrome C oxidase subunit 1 } \\ \text { CPT-1 } & \text { Carnitine palmitoyltransferase 1 } \\ \text { EDL } & \text { Extensor digitorum longus } \\ \text { HOMA-IR } & \text { HOMA index of insulin resistance } \\ \text { KD } & \text { Kinase-dead }\end{array}$




\section{Introduction}

The development of obesity-related insulin resistance is associated with chronic low-grade inflammation [1] and accumulation of bioactive lipids such as fatty acyl-CoAs, diacylglycerol and ceramide in skeletal muscle [2]. The combination of inflammation and accumulation of bioactive lipids is hypothesised to antagonise insulin signalling and insulin-stimulated glucose uptake by activating c-jun $\mathrm{N}$ terminal kinase [3], inhibitory kappa beta kinase [4], protein kinase $\mathrm{C}$ theta [5] and protein phosphatase 2A [6].

As reviewed [7], muscle lipid accumulation is due to an imbalance between myocellular lipid uptake and mitochondrial $\beta$-oxidation. In humans, skeletal muscle fatty acid oxidation is reduced with obesity [8] and type 2 diabetes [9], an effect associated with reduced mitochondrial capacity in some [10-12], but not all studies [13]. However, in animal models of obesity and insulin resistance skeletal muscle mitochondrial capacity is increased [14, 15], suggesting that despite increases in mitochondrial capacity, the oxidative rate of muscle is unable to fully compensate for increases in fatty acid delivery and uptake $[16,17]$. In accordance with this idea, increased protein levels of carnitine palmitoyltransferase 1 (CPT-1), the rate-limiting factor involved in mitochondrial uptake of long-chain carnitine acyl-CoAs, protects muscle from lipid accumulation and insulin resistance [18], whereas chronic inhibition of CPT-1 exacerbates obesityrelated insulin resistance [19]. The activity of CPT-1 is inhibited by malonyl-CoA which is produced by acetyl-CoA carboxylase (ACC) 2 and degraded by malonyl-CoA decarboxylase [20]. The importance of ACC2 is highlighted by the ACC2-null mouse, which has increased fatty acid oxidation and is protected from ectopic lipid accumulation in response to high-fat feeding [21]. The AMP-activated protein kinase (AMPK) is a well-documented ACC2 S228 kinase and AMPK-dependent phosphorylation of ACC2 reduces ACC2 activity [22] and malonyl-CoA production, in turn increasing lipid oxidation [23]. In addition to acutely regulating mitochondrial fatty acid flux, AMPK is also important in regulating mitochondrial biogenesis and oxidative capacity in skeletal muscle $[24,25]$.

In skeletal muscle from lean type 2 diabetic patients [26, 27] and obese participants [28], AMPK signalling in response to aminoimidazole carboxamide ribonucleotide (AICAR) or exercise appeared to be unchanged, although some studies have noticed modest reductions [29, 30]. Thus, it is currently not clear whether a reduction in AMPK causes accumulation of skeletal muscle lipids and insulin resistance with obesity. A recent study by Fujii et al. [31] reported that reduced muscle AMPK activity aggravated muscle insulin resistance following a high-fat diet over 30 weeks in transgenic mice on an FVB background. However, the mechanisms by which reduced muscle AMPK activity contributed to the worsening of insulin resistance with obesity were unknown, as muscle lipids were not elevated and insulin signalling not assessed. In addition, muscle insulin sensitivity was only different between genotypes 4 months after the first evidence of wholebody glucose intolerance. Thus, it is unclear whether the deficiency in muscle AMPK had directly caused insulin resistance or whether this was due to secondary effects. Therefore, the purpose of the current study was to test whether a reduction in skeletal muscle AMPK activity directly promotes ectopic lipid accumulation and insulin resistance with obesity in skeletal muscle. We used the C57/BL6 mouse strain, which is more prone than FVB mice to develop diabetes and ectopic lipid accumulation in response to high-fat feeding [32]. We hypothesised that reduced muscle AMPK activity and ACC2 phosphorylation in AMPK $\alpha 2$ kinase-dead (KD) mice [33] would exacerbate lipid accumulation and insulin resistance during high-fat feeding.

\section{Methods}

Animals We used 6- to 9-week-old male C57/BL6 (>F10) mice overexpressing an AMPK $\alpha 2 \mathrm{KD}$ transgene or their wild-type littermates in all experiments (mice were a kind gift from M. J. Birnbaum, Howard Hughes Medical Institute, Philadelphia, PA, USA). Transgenic AMPK $\alpha 2$ protein under control of the muscle creatine kinase promoter has been described previously [33]. All mice were kept on a $12 \mathrm{~h}$ light-dark cycle at $20-21^{\circ} \mathrm{C}$ and had free access to food and water unless otherwise stated. Mice were maintained on a standard rodent chow diet or low-fat diet (4\% energy from fat; Harlan Teklad) except in high-fat diet experiments, where the diet was composed of $45 \%$ energy from fat (SF04-027; Specialty Feeds, Perth, WA, Australia). These same dietary conditions were also used in separate experiments on C57/BL6j mice (Walter Eliza Hall Institute of Medical Research, Melbourne, VIC, Australia). Last, ob/ob mice (Monash Mouseworks, Melbourne, VIC, Australia) were also used to test how effectively AICAR stimulated glucose uptake. All procedures were approved by the St Vincent's Hospital Animal Ethics Committee.

Muscle incubations Soleus (slow- and fast-twitch fibres) and extensor digitorum longus (EDL) (primarily fast-twitch fibres) muscles were dissected from anaesthetised mice (6 mg of pentobarbital per $100 \mathrm{~g}$ body weight) and transferred to incubation flasks containing $2 \mathrm{ml}$ of essential buffer (Krebs-Henseleit buffer, pH 7.4, with $2.0 \mathrm{mmol} / \mathrm{l}$ pyruvate, $8 \mathrm{mmol} / 1$ mannitol and $0.1 \%$ vol BSA), gassed with $95 \%$ $\mathrm{O}_{2}+5 \% \mathrm{CO}_{2}$ and maintained at $30^{\circ} \mathrm{C}$ as described [34]. For all experiments, muscles were pre-incubated for $15 \mathrm{~min}$ in this buffer. Muscles were then incubated for an additional 20 min with a similar medium containing in 
addition either $2 \mathrm{mmol} / \mathrm{l}$ AICAR (Toronto Research Chemicals, North York, ON, Canada) or $2.8 \mu \mathrm{mol} / 1$ insulin (Actrapid; Novo Nordisk, Bagsværd, Denmark). 2-Deoxy-Dglucose uptake was measured by replacing existing incubation buffer with a similar buffer containing in addition $18.5 \mathrm{kBq} / \mathrm{ml} 2$-deoxy-D-[2,6- $\left.{ }^{3} \mathrm{H}\right]$ glucose, $1 \mathrm{mmol} / 1$ 2-deoxyD-glucose and $7.4 \mathrm{kBq}\left[1-{ }^{14} \mathrm{C}\right] \mathrm{mannitol} / \mathrm{ml}$. Measurement of 2-deoxy-D-glucose uptake was during the last $20 \mathrm{~min}$ of incubations. After preparing muscles as described below, radioactivity was measured in muscle lysates by liquid scintillation counting (Tri-Carb 2000; Packard Instruments, Meriden, CT, USA).

Intravenous insulin injection Mice were anaesthetised (6 mg of pentobarbital per $100 \mathrm{~g}$ body weight). After gently removing the gastrocnemius muscle, the inferior vena cava was exposed and insulin $(5.2 \mu \mathrm{mol} / \mathrm{kg}$ in a volume of $100 \mu \mathrm{l} / 10 \mathrm{~g}$ ) was injected using an insulin syringe. After another $5 \mathrm{~min}$ the quadriceps muscle was removed, snapfrozen and stored at $-80^{\circ} \mathrm{C}$ until analysis.

Muscle lysate preparation Muscles were homogenised in ice-cold buffer (50 mmol/1 HEPES, $\mathrm{pH} 7.4,150 \mathrm{mmol} / \mathrm{l} \mathrm{NaCl}$, $10 \mathrm{mmol} / 1 \mathrm{NaF}, 1 \mathrm{mmol} / 1$ sodium pyrophosphate, $0.5 \mathrm{mmol} /$ 1 EDTA, $250 \mathrm{mmol} / 1$ sucrose, $1 \mathrm{mmol} / 1$ dithiothreitol, $1 \%$ (vol./vol.) TritonX-100, $1 \mathrm{mmol} / 1 \mathrm{Na}_{3} \mathrm{VO}_{4}$ and 1 Roche protease inhibitor tablet per $50 \mathrm{ml}$ buffer) using an electrical homogeniser. Lysates were generated as previously described [34] and stored at $-80^{\circ} \mathrm{C}$ until analysis. Protein content in lysates was measured by the bicinchoninic acid method (Pierce, Rockford, IL, USA).

Immunoblotting Levels or phosphorylation of investigated proteins was determined in muscle lysates by SDS-PAGE and immunoblotting using the following primary antibodies: pan- $\alpha$ AMPK, phospho-AMPK T172 and phospho-ACC S228 [35]; Akt and phospho-Akt S473 (Cell Signaling Technology, MA, USA); cytochrome C oxidase subunit 1 (COX1) (Invitrogen Molecular Probes, Carlsbad, CA, USA); Complex II subunit $30 \mathrm{kDa}$, Complex III subunit Core 2, Complex IV subunit II and ATP synthase subunit alpha (OXPHOS AB cocktail; MitoSciences, , Eugene, OR, USA). Secondary antibodies were horseradishconjugated protein G (Bio-Rad Laboratories, Hercules, CA, USA). Bands were visualised using an enhanced chemoluminescence system and quantified using ImageQuant TL 05 software (Amersham Biosciences, UK). Values obtained using phospho-specific antibodies are expressed as the ratio to the total content of the protein measured after stripping the membrane and re-probing. Values obtained using antibodies against the total protein are expressed in relative units in comparison with control samples loaded on each gel.
AMPK activity AMPK $\alpha 1$ and $\alpha 2$ activities were measured from $100 \mu \mathrm{g}$ of muscle lysate protein using rabbit polyclonal AMPK antibodies for immunoprecipitation as previously described [35].

Muscle lipids Muscle triacylglycerol, ceramide and diacylyglyerol were extracted from freeze-dried powdered gastrocnemius muscle and measured as previously described [36].

Insulin and glucose tolerance test Tolerance tests were performed on AMPK KD and wild-type mice after 6, 10 and 11 weeks of high-fat feeding. Glucose $(1 \mathrm{~g} / \mathrm{kg})$ or insulin $(5.2 \mu \mathrm{mol} / \mathrm{kg}$; Actrapid, Novo Nordisk) in saline was given intraperitoneally and tail blood glucose was determined using a glucometer (Bayer, Leverkusen, Germany) [37]. Mice were fasted for $6 \mathrm{~h}$ starting at 08:00 hours before all tests.

Serum insulin and leptin Insulin concentrations were measured in serum from mice in the fed state, after $6 \mathrm{~h}$ fasting and in fasted mice $20 \mathrm{~min}$ after an i.p. glucose injection (1 $\mathrm{g} / \mathrm{kg}$ ) using an ELISA kit (Millipore, Linco Research, Billerica, MA, USA) according to the manufacturer's recommendations. Leptin was measured in serum from fed mice using a mouse adipokine kit (Linco Research) and a workstation (Bio-plex 200; Bio-Rad Laboratories,) as described [38].

HOMA index of insulin resistance The HOMA index of insulin resistance (HOMA-IR) score was determined as: fasting serum insulin $(\mathrm{pmol} / \mathrm{ml}) \times$ fasting plasma glucose $(\mathrm{mmol} / \mathrm{l}) / 156$, and is a measure of whole-body insulin sensitivity [39].

Statistics Data are expressed as means \pm SE. Statistical evaluations were performed by Student's $t$ test or two-way ANOVA using the Student-Newman-Keuls method as a post hoc test when appropriate. Differences between groups were considered statistically significant if $p<0.05$.

\section{Results}

Muscle AMPK activity and ACC phosphorylation In agreement with previous reports [33, 40, 41], $\alpha 2 \mathrm{AMPK}$ activity was reduced in the gastrocnemius $(\sim 50 \%)$, soleus $(\sim 80 \%)$ and EDL $(\sim 95 \%)$ muscles from KD mice compared with wild-type (Fig. 1a,c). $\alpha 1$ AMPK activity was not significantly reduced in gastrocnemius muscles, but was reduced by $\sim 45 \%$ in soleus and EDL muscles from KD mice (Fig. 1a,c). AMPK regulates muscles lipid oxidation 


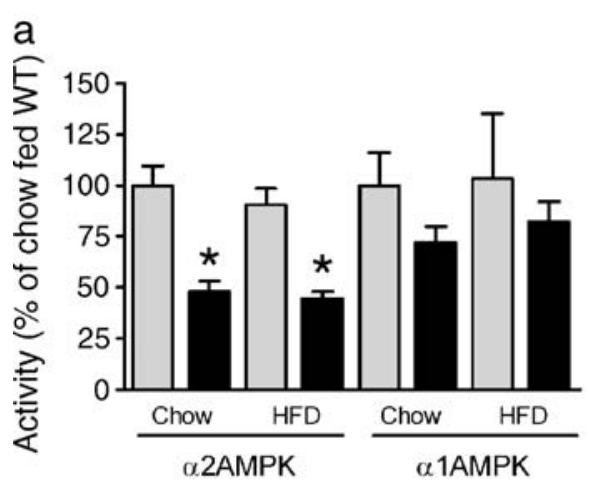

b

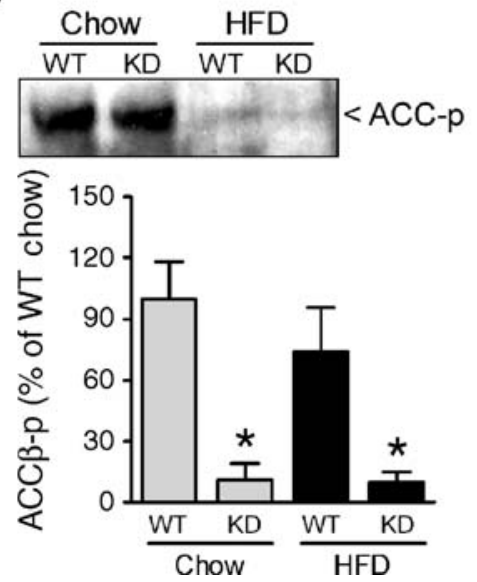

C

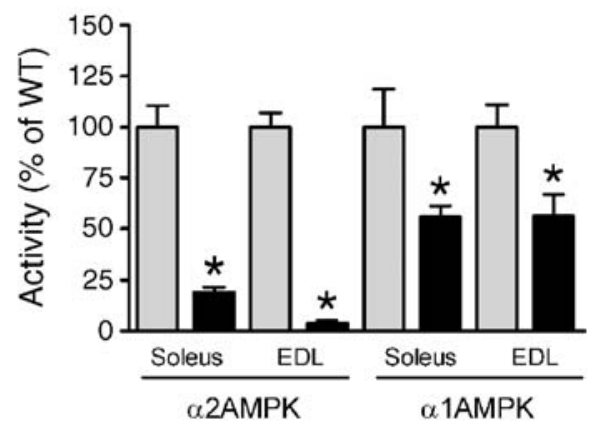

Fig. 1 AMPK activity and ACC2 phosphorylation. a Gastrocnemius $\alpha 2$ and $\alpha 1$ AMPK activity and b ACC2 S228 phosphorylation and representative immunoblot from AMPK KD mice and wild-type (WT) littermates fed a chow or a high-fat diet (HFD). $\mathbf{c} \alpha 2$ and $\alpha 1$ AMPK activity in soleus and EDl muscle from chow-fed AMPK KD mice and wild-type littermates. Black bars (a, c), KD; grey bars, wild-type. Values are mean \pm SEM (two-way ANOVA); ${ }^{*} p<0.05$ for difference from wild-type, $n=8-11$ through phosphorylation and deactivation of ACC2 [22, 40, 41]. As previously demonstrated [33, 40, 41], ACC2 serine 228 phosphorylation was reduced by $\sim 90 \%$ in $\mathrm{KD}$ gastrocnemius muscle (Fig. 1c). The 12 weeks of high-fat diet did not alter AMPK $\alpha 1$ and $\alpha 2$ activities or ACC2 phosphorylation (Fig. 1a,b).

Body weight, obesity and serum non-esterified fatty acid High-fat feeding for 12 weeks increased body mass by $\sim 40 \%$ and epididymal fat pad weight by $\sim 330 \%$ in wildtype and $\mathrm{KD}$ mice compared with chow-fed controls; however, these increases were not different between genotypes (Fig. 2a,b). The high-fat diet increased fasting serum levels of NEFA by $\sim 35 \%$, this increase being similar between the two genotypes (Fig. 2c).
Serum glucose, insulin and leptin, and HOMA-IR Fasting serum glucose was similar in chow-fed wild-type and KD mice, and was increased in response to high-fat feeding by $\sim 35 \%$ in both genotypes (Table 1). In chow-fed mice, serum insulin during fasting, in the fed state and in response to a glucose injection was similar in wild-type and KD mice (Table 1). High-fat feeding increased serum insulin in the three conditions by 5.4-, 11- and 17-fold, respectively, compared with corresponding chow-fed mice (Table 1), demonstrating a significant degree of hyperinsulinaemia with obesity. Unexpectedly, in the fed state, the degree of hyperinsulinaemia was lower in high-fat-fed KD mice than wild-type littermates, but this was not evident in the fasted state or $20 \mathrm{~min}$ post glucose injection (Table 1). The HOMA-IR showed a significant increase in

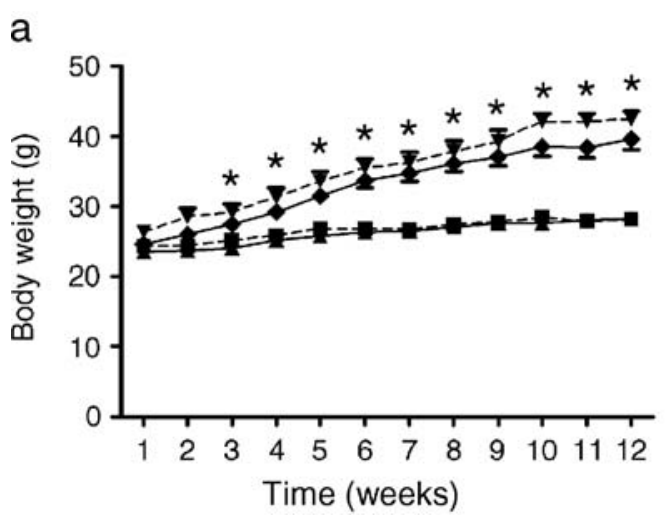

Fig. 2 Body weight, adiposity and NEFA. a Weekly body weights of AMPK KD mice fed a chow (black triangles) or a high-fat ( $45 \%$ energy from fat) (black diamonds) diet for 12 weeks and those of wild-type littermates (black squares, chow diet; inverted triangles, high-fat diet). b Epididymal fat pat weight of AMPK KD mice and wild-type littermates b

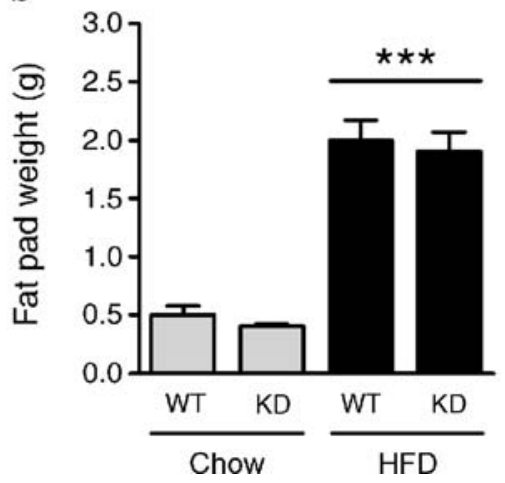

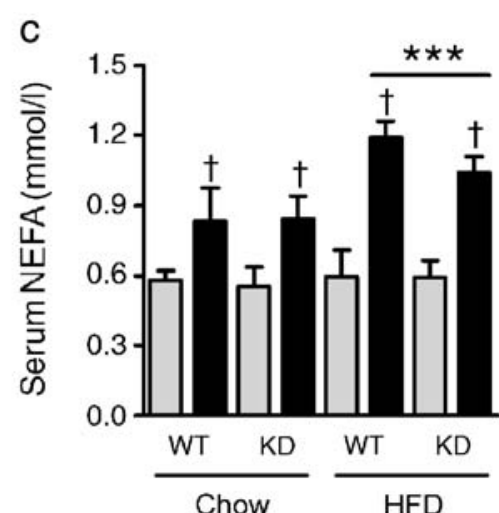

after 12 weeks of chow or HF diet (HFD) feeding. c Serum NEFA of fed (grey bars) and $6 \mathrm{~h}$ fasted (black bars) chow or HFD fed AMPK KD mice and wild-type littermates. Values are mean \pm SEM (two-way ANOVA). ${ }^{\dagger} p<0.05$ for difference from fed mice; ${ }^{*} p<0.05$ and $* * * p<$ 0.001 for difference from chow-fed; $n=8-12$ 
Table 1 Metabolic variables from chow- and high-fat diet-fed wild-type and AMPK KD mice

Values are mean \pm SEM, $n=8-12$

${ }^{a}$ Levels measured in serum ${ }^{*} p<0.05$ for difference from chow-fed in same genotype; ${ }^{\dagger} p<0.05$ for difference from $\mathrm{KD}$ in same intervention group;

WT, wild-type

\begin{tabular}{|c|c|c|c|c|c|}
\hline \multirow[t]{2}{*}{ Variables } & \multirow[t]{2}{*}{ Units } & \multicolumn{2}{|l|}{ Chow } & \multicolumn{2}{|l|}{ High-fat diet } \\
\hline & & WT & $\mathrm{KD}$ & WT & $\mathrm{KD}$ \\
\hline Glucose, fasted $^{\mathrm{a}}$ & $\mathrm{mmol} / \mathrm{l}$ & $9.6 \pm 0.3$ & $9.4 \pm 0.4$ & $13.1 \pm 0.4^{*}$ & $12.6 \pm 0.5^{*}$ \\
\hline Insulin, fasted ${ }^{\mathrm{a}}$ & $\mathrm{pmol} / 1$ & $43 \pm 7$ & $53 \pm 12$ & $234 \pm 45^{*}$ & $234 \pm 59^{*}$ \\
\hline Insulin, fed ${ }^{\mathrm{a}}$ & $\mathrm{pmol} / 1$ & $74 \pm 15$ & $60 \pm 3$ & $777 \pm 110^{*}$ & $410 \pm 71 * \dagger$ \\
\hline Insulin, GTT ${ }^{\mathrm{a}}$ & $\mathrm{pmol} / 1$ & $38 \pm 3$ & $69 \pm 10$ & $639 \pm 167 *$ & $467 \pm 077^{*}$ \\
\hline Leptin $^{\mathrm{a}}$ & $\mathrm{pmol} / 1$ & $146 \pm 50$ & $169 \pm 45$ & $775 \pm 39 *$ & $925 \pm 231^{*}$ \\
\hline HOMA-IR & Index & $3.5 \pm 0.6$ & $3.7 \pm 0.8$ & $23.3 \pm 3.9^{*}$ & $22.8 \pm 6.3^{*}$ \\
\hline
\end{tabular}

HOMA-IR with obesity and no difference in this index between genotypes (Table 1). Finally, the high-fat diet induced a $\sim$ fivefold increase in serum leptin compared with chow-fed controls, this increase being similar in wild-type and KD mice (Table 1). These results show that obese KD mice do not develop a more severe degree of hyperinsulinaemia, hyperglycaemia and hyperleptinaemia than obese wild-type littermates.

Glucose and insulin tolerance test Whole-body glucose tolerance was tested after 6 and 11 weeks of high-fat feeding. Glucose tolerance was impaired at both time points, an effect which was more pronounced after 11 than 6 weeks on the high-fat diet (Fig. 3a,b). Surprisingly, this progression in glucose intolerance was similar between wild-type and KD mice (Fig. 3a,b). In addition, 10 weeks of high-fat feeding reduced whole-body insulin sensitivity, again with no differences between genotypes (Fig. 3c).

Insulin-stimulated glucose uptake and Akt phosphorylation To directly assess muscle insulin action, we isolated soleus and EDL muscles from mice fed the high-fat diet for
12 weeks and incubated them with a sub-maximal concentration of insulin. Insulin increased glucose uptake in the soleus and EDL muscle by two- and threefold, respectively (Fig. 4a,b), with similar increases in chow-fed wild-type and KD muscles (Fig. 4a,b). As anticipated, the high-fat diet in wild-type mice reduced insulin-stimulated glucose uptake by $\sim 40 \%$ in the soleus and by $\sim 50 \%$ in the EDL muscles. However, this reduction in insulin-stimulated glucose uptake was not exacerbated in muscle from AMPK KD mice (Fig. 4a,b). Phosphorylation of the insulin signalling protein Akt at S473 reflected a similar pattern to the glucose uptake measures, with the high-fat diet suppressing insulin-induced phosphorylation of Akt by 50 to $60 \%$ in muscle from wild-type and KD mice (Fig. 4c,d). To observe whether the degree of insulin resistance was also similar in vivo, we injected mice intravenously with an insulin bolus at the same concentration used during the insulin tolerance tests and measured Akt phosphorylation in the quadriceps muscle. Insulin increased Akt phosphorylation in both genotypes (Fig. 4e), an effect which was suppressed by $\sim 50 \%$, irrespective of genotype, in animals fed a high-fat diet. These data indicate that, surprisingly,

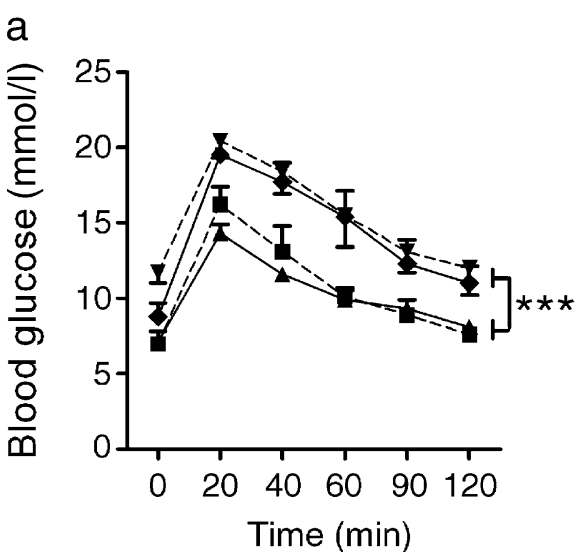

Fig. 3 Whole-body glucose tolerance and insulin tolerance tests. a Whole-body glucose tolerance at week 6 and (b) week 11, and (c) insulin tolerance at week 10 of AMPK KD mice fed chow (black triangles) or a high-fat (black diamonds) diet and of wild-type littermates
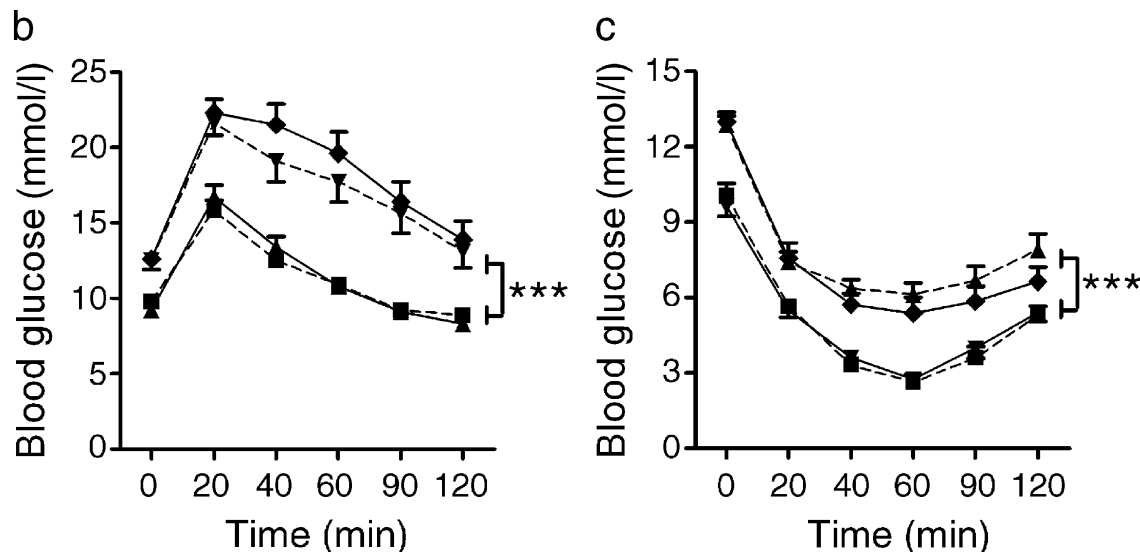

(black squares, chow diet; inverted triangles, high-fat diet). Glucose load $1 \mathrm{~g} / \mathrm{kg}$; insulin infusion $5.2 \mu \mathrm{mol} / \mathrm{kg}$. Values are mean \pm SEM (two-way ANOVA). ${ }^{* *} p<0.001$ for difference from chow-fed; $n=8-12$ 
a

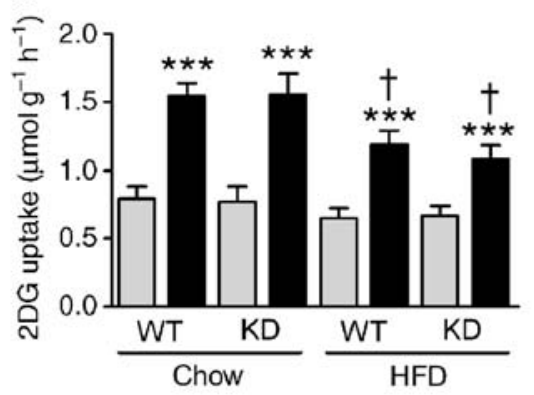

b

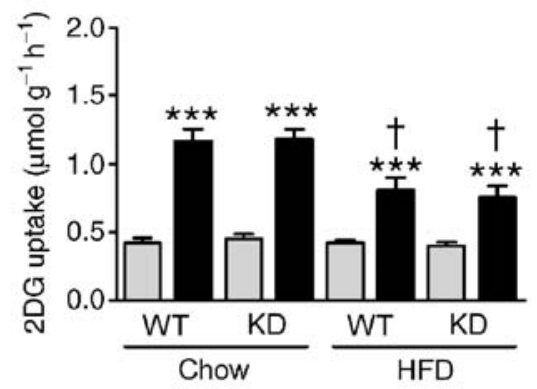

C
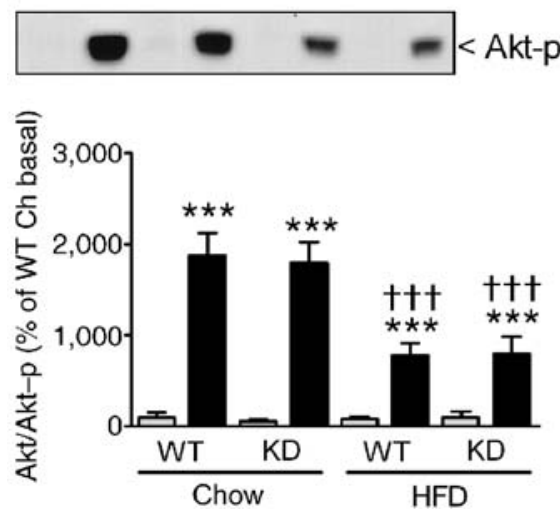

Fig. 4 Insulin-stimulated glucose uptake and Akt phosphorylation with representative immunoblots after 12 weeks of chow $(\mathrm{Ch})$ or highfat diet (HFD). Basal (grey bars) and insulin-stimulated (black bars) glucose uptake was measured in incubated soleus (a) and EDL (b) muscles from chow and high-fat diet-fed AMPK KD mice and wildtype littermates. c Basal and insulin-stimulated S473 Akt phosphor-

KD mice did not develop greater (or more severe) muscle insulin resistance than wild-type littermates with obesity in response to a short-term high fat diet.

Muscle lipids Diacylglycerol [42, 43] and ceramides [44, 45] have been implicated in obesity-associated insulin resistance. Here, high-fat feeding increased gastrocnemius muscle triacylglycerol and ceramides by 300 and $50 \%$, respectively, with a trend $(p=0.07)$ to increased diacylglycerol content also observed (Fig. 5a-c). These increases were similar in wild-type and KD muscles. Thus, accumulation of lipid species that impair insulin signalling was not exacerbated in AMPK KD muscles after 12 weeks of high-fat feeding.

Muscle mitochondrial markers Previous studies have found that AMPK regulates mitochondrial biogenesis [24, 25] and that high-fat feeding increases mitochondrial oxidative capacity $[14,15]$. To estimate mitochondrial content, we measured levels of mitochondrial marker proteins in gastrocnemius muscle (Fig. 6). In chow-fed KD muscles, protein levels of the complex II Ip subunit tended to be reduced by $\sim 20 \%(p=0.10)$, while the complex III Core 2 subunit was significantly reduced by $40 \%$ compared with e Akt-p
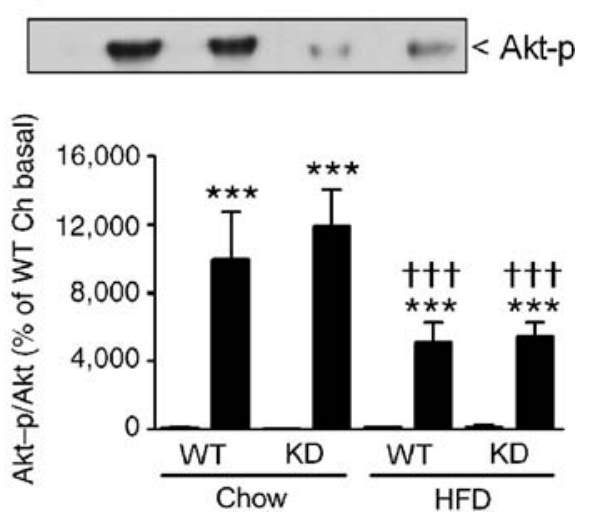

ylation was measured in lysates from incubated soleus and (d) EDL muscle from groups as above (a,b). e S473 Akt phosphorylation in gastrocnemius (basal) and quadriceps (insulin) muscle from mice injected intravenously with insulin. Values are mean \pm SEM (two-way ANOVA). $* * * p<0.001$ for difference from basal; ${ }^{\dagger} p<0.05$ and ${ }^{\dagger \dagger \dagger} p<$ 0.001 for difference from chow-fed; $n=8-12$

wild-type littermates (Fig. 6). High-fat feeding was associated with increased levels of mitochondrial proteins regardless of genotype, these increases being particularly evident for the complex III subunits Core 2 and complex IV subunit COX-I (Fig. 6). Thus, while a modest reduction in some mitochondrial markers was detected in chow-fed KD mice, there was no difference in levels of mitochondrial markers after 12 weeks of high-fat feeding between the two genotypes.

Effect of obesity on AMPK phosphorylation and AICARstimulated glucose uptake To further investigate a potential role of AMPK in obesity-related insulin resistance, we assessed whether the ability of the AMPK activator AICAR to increase glucose uptake was impaired in muscles from high-fat-fed wild-type C57/BL6 and ob/ob mice. In high-fat diet muscles, AICAR increased glucose uptake in soleus and EDL by 70 and $200 \%$, respectively; these increases were not affected by high-fat feeding (Fig. 7a,b). In agreement with normal activation of AICAR-stimulated glucose uptake, activating phosphorylation of $\alpha$ AMPK T172 in response to AICAR increased normally in muscle from obese mice fed a high-fat diet (Fig. 7c). Since we have 
Fig. 5 Muscle lipids in gastrocnemius muscle after a 12 week chow or high-fat diet (HFD). a Triacylglycerol, (b) diacylglycerol and (c) ceramides in muscle from chow and HFD-fed AMPK $\mathrm{KD}$ mice and wild-type littermates. Values are mean \pm SEM (two-way ANOVA). *** $p<0.001$ for difference from chow-fed; ${ }^{\dagger} p=0.07 ; n=8-12$. DM, dry matter
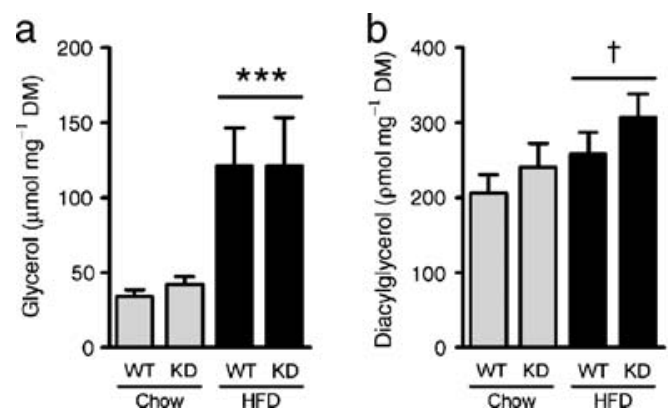

previously reported that $\mathrm{TNF} \alpha$ impairs AMPK signalling in muscles from the $o b / o b$ mouse [36], we also tested the effectiveness with which AICAR increases glucose uptake in this more severe model of obesity. We found that AICAR-stimulated glucose uptake was ameliorated in soleus and substantially reduced in EDL from $o b / o b$ mice (Fig. 7d). Taken together, these data suggest that obesity in response to high-fat feeding is not associated with impaired AMPK actions; however, in the case of severe obesity and insulin resistance, as seen in the $o b / o b$ mouse, defects in AMPK signalling may exacerbate this phenotype.

\section{a}

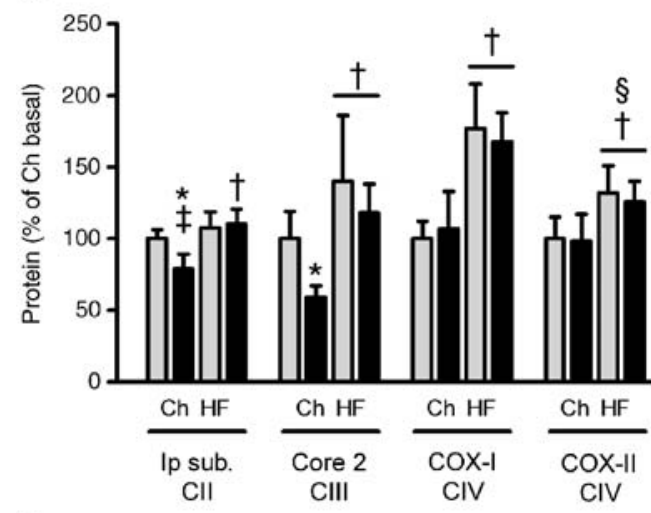

b

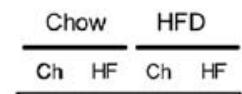

Ip sub

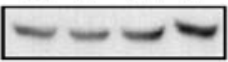

Core 2

COX-1

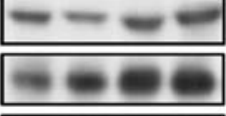

COX-11

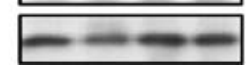

Fig. 6 Protein content of mitochondrial markers. a Ip subunit complex II, Core 2 subunit complex III, COX-I complex IV and COXII complex IV were quantified after measurement by (b) immunoblot in gastrocnemius muscle lysates from AMPK KD mice (black bars) and wild-type littermates (grey bars) after 12 weeks of chow (Ch) or high-fat (HF) diet. Values are mean \pm SEM (two-way ANOVA). ${ }^{*} p<0.05,{ }^{\star} p=$ 0.10 for difference from wild-type; ${ }^{\dagger} p<0.05,{ }^{\S} p=0.09$ for difference from chow-fed; $n=8-12$

\section{Discussion}

The accumulation of diacylglycerol and ceramides is associated with insulin resistance in skeletal muscle [4245]. As AMPK is thought to control fatty acid oxidation via the $\mathrm{ACC} 2 \rightarrow$ malonyl-CoA $\rightarrow$ CPT-1 axis $[20,46]$ and activation of this pathway prevents lipid-induced insulin resistance [38, 47-49], we investigated whether a deficiency in muscle AMPK would exacerbate obesity-related insulin resistance due to increased accumulation of bioactive lipid species. We used a mouse model specifically overexpressing a KD AMPK $\alpha 2$ in skeletal and heart muscle and characterised by a substantial reduction in ACC2 phosphorylation [40]. We assessed the efficacy with which insulin increased Akt phosphorylation and glucose uptake after 12 weeks of a high-fat diet and found, somewhat unexpectedly, that muscles from KD mice develop insulin resistance to a similar degree to their wild-type littermates and thus do not become more insulinresistant with obesity as initially hypothesised. In accordance, whole-body glucose and insulin intolerance were impaired to the same degree with high-fat feeding in wildtype and KD mice. As high-fat feeding in wild-type and KD mice was associated with comparable increases in muscle lipids, these data suggest that a significant reduction in ACC2 phosphorylation does not translate into increased muscle lipid storage and that a reduction in muscle AMPK does not make the muscle more susceptible to high-fat dietor obesity-induced insulin resistance.

In the present study we found a $90 \%$ reduction in ACC phosphorylation in $\mathrm{KD}$ mouse muscle. These findings are in agreement with previous studies [38, 40, 41] and suggest that residual $\alpha 1$ AMPK activity is unable to compensate for the reduced $\alpha 2 \mathrm{AMPK}$ activity when it comes to ACC phosphorylation. Surprisingly, this dramatic reduction in resting/basal ACC phosphorylation in $\mathrm{KD}$ mice did not result in greater accumulation of muscle lipids or increases in body mass, epididymal fat pad weight or serum leptin between high-fat fed KD and wild-type mice. These findings suggest that lipid metabolism was not altered in the obese KD mouse and are in line with recent reports showing that basal fatty acid oxidation is not reduced in 
Fig. 7 AICAR-stimulated glucose uptake in high-fat diet (HFD) fed and $o b / o b$ mice. AICAR and insulin-stimulated glucose uptake was measured in incubated soleus (a) and EDL (b) muscles from chow (Ch) or HFD fed C57/BL6 mice. c Basal and AICAR-stimulated AMPK T172 phosphorylation was measured in lysates from incubated EDL and soleus muscles from chow (grey bars) or HFD (black bars) fed C57/BL6 mice. d Basal (grey bars) and AICAR-stimulated (black bars) glucose uptake was measured in incubated soleus and EDL muscles from obese $o b / o b$ mice and lean littermates. Values are mean \pm SEM (two-way ANOVA). ${ }^{*} p<0.05$ and $* * * p<0.001$ for difference from basal; ${ }^{\dagger} p<0.05$ for difference from chow-fed; $n=8-10$. 2-DG, 2-deoxy-glucose a

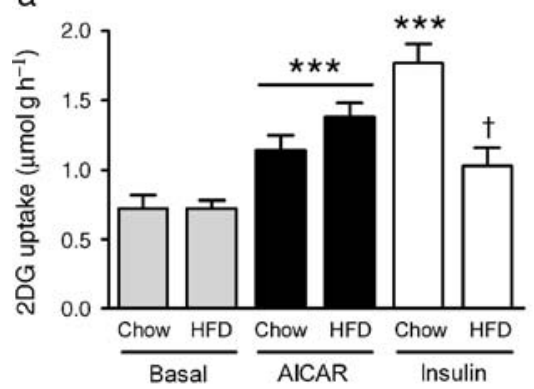

C

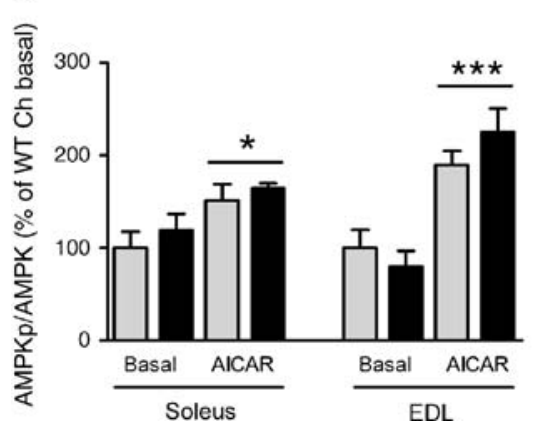

b

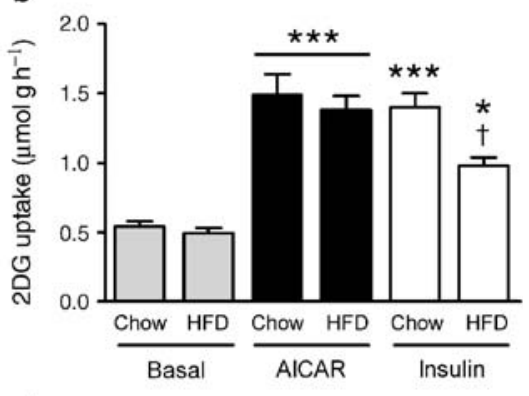

d

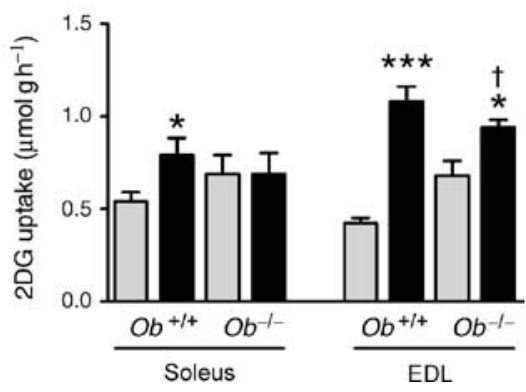

muscles from the KD mouse [40]. Taken together, these findings suggest that AMPK is not essential for regulating basal fatty acid oxidation. Furthermore, as the substantial reduction in ACC2 phosphorylation did not translate into differences in lipid accumulation with obesity, this may suggest either that ACC2 is not critical for regulating basal fatty acid oxidation in resting muscle or that only a very small amount of ACC2 phosphorylation is sufficient for maintenance of basal fatty acid oxidation.

It is conceivable that congenital deficiency in muscle AMPK induced compensatory increases in oxidative capacity to overcome the reduction in ACC2 phosphorylation. However, studies of the AMPK $\alpha 2$-null mouse have reported that CPT-1 and other mitochondrial proteins are actually reduced in AMPK-deficient muscle $[25,50]$. In agreement with these observations, we found that the content of some mitochondrial proteins was reduced in muscle from chow-fed KD mice, but that the high-fat diet increased mitochondrial protein levels to a similar degree in $\mathrm{KD}$ and wild-type mice. Thus, the observation that KD muscles were able to adapt to the increased lipid load induced by a high-fat diet or obesity may in part explain the similar degrees of lipid accumulation and insulin resistance between the two genotypes.

We found that the impaired ability of insulin to stimulate glucose uptake ex vivo and to increase Akt phosphorylation in vivo and ex vivo was not exacerbated in KD muscle with obesity. In addition, we assessed whole-body insulin sensitivity by performing glucose and insulin tolerance tests and by calculating the HOMA-IR index from fasting insulin and glucose. We found that these measures were affected similarly in the two genotypes with obesity. In fact, the only genotype difference detected was a modest, but significant reduction in circulating insulin levels in fed obese KD mice, which, if anything, suggests improved insulin sensitivity at the whole-body level. These findings clearly demonstrate that impairments in insulin action were not worse in $\mathrm{KD}$ than in wild-type muscles with high-fat feeding, and are in agreement with similar increases in intramuscular lipids in the two genotypes (Fig. 5). Thus, a reduction in muscle AMPK does not affect the progression of obesity-associated insulin resistance in skeletal muscle or at the whole-body level. Therefore reduced levels of muscle AMPK activity seem unlikely to be a key contributor to the development of obesity-related insulin resistance, at least in the early phase of disease progression.

It could be argued that if AMPK is instrumental in the onset of obesity-associated insulin resistance, AMPK signalling by itself should be hampered by high-fat feeding. However, high-fat diet-induced obesity did not alter basal AMPK activity or ACC phosphorylation. Moreover, in agreement with findings in ex vivo incubated muscle strips from insulin-resistant humans [27, 28], AICARstimulated $\alpha$ AMPK-phosphorylation and glucose uptake were normal in muscles from high-fat fed mice. However, in accordance with our previous finding of suppressed AMPK signalling in this mouse model [36], we did find that AICARstimulated glucose uptake was suppressed in muscles from $o b / o b$ mice, a genetic model of severe obesity and insulin resistance. Thus, while AMPK function is impaired with severe obesity it does not appear to influence the development of insulin resistance seen in more moderate levels of obesity. 
While this manuscript was in preparation, Fujii et al. [31] demonstrated that mice on a FVB background overexpressing a muscle-specific KD AMPK $\alpha 2$ Asp157Ala mutation develop more severe muscle insulin resistance after 30 weeks on a high-fat diet with $60 \%$ of energy from fat (compared with 12 weeks of a high-fat diet with $45 \%$ of energy from fat, as used in the current study), an effect which occurred independently of increases in muscle lipids. The difference between our findings and those of Fujii et al. [31] may be related to the use of different diet protocols, but probably more importantly, to the use of different mouse strains, as the FVB strain of mice is known to be resistant to the effects of high-fat diet-induced obesity compared with mice on a C57/BL6 background [32] as used in the current study. But the fact that the genotype effect occurred 26 weeks later than the first evidence of glucose intolerance suggests that AMPK did not have a primary role in the development of insulin resistance, as was recognised by Fujii el al. [31].

In conclusion, a muscle-specific reduction in AMPK $\alpha 2$ and ACC phosphorylation did not exacerbate muscle lipid accumulation and insulin resistance in response to a shortterm diet intervention, suggesting that muscle AMPK is not a determining factor in the initial development of insulin resistance. The finding that lipid accumulation was not greater in obese AMPK $\alpha 2 \mathrm{KD}$ muscles than in wild-type littermates may in part be explained by similar expression of mitochondrial proteins in the two genotypes when fed a high-fat diet. While these data argue against a primary role for muscle AMPK in the initial development of obesity-related insulin resistance, the finding that AMPK signalling towards glucose uptake was intact in a physiological model of obesity (high-fat diet) nevertheless underscores muscle AMPK as a promising target in the therapy of type 2 diabetes and obesity-related insulin resistance.

Acknowledgements These studies were supported by grants and research fellowships from the National Health and Medical Research Council, Australia (to G. R. Steinberg, S. Beck Jøgensen and B. E. Kemp). S. Beck Jørgensen was supported by a Danish Research Council of Health and Diseases postdoctoral fellowship. G. R. Steinberg is a Canadian Research Chair in Metabolism, Obesity and Type 2 diabetes.

Duality of interest The authors declare that there is no dually of interest associated with this manuscript.

\section{References}

1. Hotamisligil GS (2006) Inflammation and metabolic disorders. Nature 444:860-867

2. Savage DB, Petersen KF, Shulman GI (2007) Disordered lipid metabolism and the pathogenesis of insulin resistance. Physiol Rev 87:507-520
3. Hirosumi J, Tuncman G, Chang L et al (2002) A central role for JNK in obesity and insulin resistance. Nature 420:333-336

4. Yuan M, Konstantopoulos N, Lee J et al (2001) Reversal of obesity- and diet-induced insulin resistance with salicylates or targeted disruption of Ikkbeta. Science 293:1673-1677

5. Kim JK, Fillmore JJ, Sunshine MJ et al (2004) PKC- $\theta$ knockout mice are protected from fat-induced insulin resistance. J Clin Invest 114:823-827

6. Cazzolli R, Carpenter L, Biden TJ, Schmitz-Peiffer C (2001) A role for protein phosphatase 2A-like activity, but not atypical protein kinase $\mathrm{C} \zeta$, in the inhibition of protein kinase $\mathrm{B} / \mathrm{Akt}$ and glycogen synthesis by palmitate. Diabetes 50:2210-2218

7. Watt MJ, Steinberg GR (2007) Pathways involved in lipidinduced insulin resistance in obesity. Future Lipidol 2:659-667

8. Kelley DE, Goodpaster B, Wing RR, Simoneau J-A (1999) Skeletal muscle fatty acid metabolism in association with insulin resistance, obesity, and weight loss. Am J Physiol 277:E1130-E1141

9. Kelley DE, Simoneau JA (1994) Impaired free fatty acid utilization by skeletal muscle in non-insulin-dependent diabetes mellitus. J Clin Invest 94:2349-2356

10. Simoneau J-A, Veerkamp JH, Turcotte LP, Kelley DE (1999) Markers of capacity to utilize fatty acids in human skeletal muscle: relation to insulin resistance and obesity and effects of weight loss. FASEB J 13:2051-2060

11. Befroy DE, Petersen KF, Dufour S et al (2007) Impaired mitochondrial substrate oxidation in muscle of insulin-resistant offspring of type 2 diabetic patients. Diabetes 56:1376-1381

12. Ritov VB, Menshikova EV, He J, Ferrell RE, Goodpaster BH, de Kelley (2005) Deficiency of subsarcolemmal mitochondria in obesity and type 2 diabetes. Diabetes $54: 8-14$

13. Boushel R, Gnaiger E, Schjerling P, Skovbro M, Kraunsoe R, Dela F (2007) Patients with type 2 diabetes have normal mitochondrial function in skeletal muscle. Diabetologia 50:790-796

14. Turner N, Bruce CR, Beale SM et al (2007) Excess lipid availability increases mitochondrial fatty acid oxidative capacity in muscle: evidence against a role for reduced fatty acid oxidation in lipid-induced insulin resistance in rodents. Diabetes 56:20852092

15. Hancock CR, Han DH, Chen M et al (2008) High-fat diets cause insulin resistance despite an increase in muscle mitochondria. Proc Natl Acad Sci U S A 105:7815-7820

16. Steinberg GR, Parolin ML, Heigenhauser GJ, Dyck DJ (2002) Leptin increases FA oxidation in lean but not obese human skeletal muscle: evidence of peripheral leptin resistance. Am J Physiol Endocrinol Metab 283:E187-E192

17. Hegarty BD, Cooney GJ, Kraegen EW, Furler SM (2002) Increased efficiency of fatty acid uptake contributes to lipid accumulation in skeletal msucle of high fat-fed insulin resistant rats. Diabetes 51:1477-1488

18. Bruce CR, Hoy AJ, Turner N et al (2009) Overexpression of carnitine palmitoyltransferase-1 in skeletal muscle is sufficient to enhance fatty acid oxidation and improve high-fat diet-induced insulin resistance. Diabetes 58:550-558

19. Dobbins RL, Szczepaniak LS, Bentley B, Esser V, Myhill J, McGarry JD (2001) Prolonged inhibition of muscle carnitine palmitoyltransferase-1 promotes intramyocellular lipid accumulation and insulin resistance in rats. Diabetes 50:123-130

20. Ruderman NB, Saha AK, Vavvas D, Witters LA (1999) MalonylCoA, fuel sensing, and insulin resistance. Am J Physiol 276:E1E18

21. Abu-Elheiga L, Matzuk MM, Abo-Hashema KA, Wakil SJ (2001) Continuous fatty acid oxidation and reduced fat storage in mice lacking acetyl-CoA carboxylase 2. Science 291:2613-2616

22. Davies SP, Sim AT, Hardie DG (1990) Location and function of three sites phosphorylated on rat acetyl-CoA carboxylase by the AMP-activated protein kinase. Eur J Biochem 187:183-190 
23. Merrill GF, Kurth EJ, Hardie DG, Winder WW (1997) AICA riboside increases AMP-activated protein kinase, fatty acid oxidation, and glucose uptake in rat muscle. Am J Physiol 273:E1107-E1112

24. Zong H, Ren JM, Young LH et al (2002) AMP kinase is required for mitochondrial biogenesis in skeletal muscle in response to chronic energy deprivation. Proc Natl Acad Sci U S A 99:15983-15987

25. Jorgensen SB, Treebak JT, Viollet B et al (2006) Role of $\alpha 2$-AMPK in basal, training- and AICAR-induced GLUT4, hexokinase II and mitochondrial protein expression in mouse muscle. Am J Physiol Endocrinol Metab 292:E331-E339

26. Musi N, Fujii N, Hirshman MF et al (2001) AMP-activated protein kinase (AMPK) is activated in muscle of subjects with type 2 diabetes during exercise. Diabetes 50:921-927

27. Koistinen HA, Galuska D, Chibalin AV et al (2003) 5-Aminoimidazole carboxamide riboside increases glucose transport and cell-surface GLUT4 content in skeletal muscle from subjects with type 2 diabetes. Diabetes 52:1066-1072

28. Steinberg GR, Smith AC, van Denderen BJW et al (2004) AMPactivated protein kinase is not down-regulated in human skeletal muscle of obese females. J Clin Endocrinol Metab 89:4575-4580

29. Sriwijitkamol A, Coletta DK, Wajcberg E et al (2007) Effect of acute exercise on AMPK signaling in skeletal muscle of subjects with type 2 diabetes: a time-course and dose-response study. Diabetes 56:836-848

30. Bandyopadhyay GK, Yu JG, Ofrecio J, Olefsky JM (2006) Increased malonyl-CoA levels in muscle from obese and type 2 diabetic subjects lead to decreased fatty acid oxidation and increased lipogenesis; thiazolidinedione treatment reverses these defects. Diabetes 55:2277-2285

31. Fujii N, Ho RC, Manabe Y et al (2008) Ablation of AMPactivated protein kinase $\alpha 2$ activity exacerbates insulin resistance induced by high-fat feeding of mice. Diabetes 57:2958-2966

32. Hu CC, Qing K, Chen Y (2004) Diet-induced changes in stearoylCoA desaturase 1 expression in obesity-prone and -resistant mice. Obes Res 12:1264-1270

33. Mu J, Brozinick JT Jr, Valladares O, Bucan M, Birnbaum MJ (2001) A role for AMP-activated protein kinase in contractionand hypoxia-regulated glucose transport in skeletal muscle. Mol Cell 7:1085-1094

34. Jorgensen SB, Oakhill JS, Fazakerley D, Stoeckli J, Kemp BE, Steinberg GR (2009) Oligomeric resistin impairs insulin and AICAR-stimulated glucose uptake in mouse skeletal muscle by inhibiting GLUT4 translocation. Am J Physiol Endocrinol Metab 297:E57-E66

35. Chen X-P, McConell GK, Michell BJ, Snow RJ, Canny BJ, Kemp BE (2000) AMPK signaling in contracting human skeletal muscle: aceyl-CoA carboxylase and NO synthase phosphorylation. Am J Physiol 279:E1202-E1206

36. Steinberg GR, Michell BJ, van Denderen BJ et al (2006) Tumor necrosis factor alpha-induced skeletal muscle insulin resistance involves suppression of AMP-kinase signaling. Cell Metab 4:465-474
37. Jorgensen SB, Viollet B, Andreelli F et al (2004) Knockout of the alpha2 but not alpha1 5'-AMP-activated protein kinase isoform abolishes 5-aminoimidazole-4-carboxamide-1-beta-4-ribofuranosidebut not contraction-induced glucose uptake in skeletal muscle. J Biol Chem 279:1070-1079

38. Watt MJ, Dzamko N, THomas WG et al (2006) CNTF reverses obesity-induced insulin resistance by activating skeletal muscle AMPK. Nat Med 12:541-548

39. Matthews DR, Hosker JP, Rudenski AS, Naylor BA, Treacher DF, Turner RC (1985) Homeostasis model assessment: insulin resistance and beta-cell function from fasting plasma glucose and insulin concentrations in man. Diabetologia 28:412-419

40. Dzamko NL, Schertzer JD, Ryall J et al (2008) AMPK independent pathways regulate skeletal muscle fatty acid oxidation. J Physiol 586:5819-5831

41. Steinberg GR, Watt MJ, Ernst M, Birnbaum MJ, Kemp BE, Jorgensen SB (2009) Ciliary neurotrophic factor stimulates muscle glucose uptake by a PI3-kinase-dependent pathway that is impaired with obesity. Diabetes 58:829-839

42. Turinsky J, O'Sullivan DM, Bayly BP (1990) 1, 2-Diacylglycerol and ceramide levels in insulin-resistant tissues of the rat in vivo. $\mathrm{J}$ Biol Chem 265:16880-16885

43. Yu C, Chen Y, Cline GW et al (2002) Mechanism by which fatty acids inhibit insulin activation of insulin receptor substrate-1 (IRS-1)-associated phosphatidylinositol 3-kinase activity in muscle. J Biol Chem 277:50230-50236

44. Holland WL, Brozinick JT, Wang LP et al (2007) Inhibition of ceramide synthesis ameliorates glucocorticoid-, saturated-fat-, and obesity-induced insulin resistance. Cell Metab 5:167-179

45. Schmitz-Peiffer C, Craig DL, Biden TJ (1999) Ceramide generation is sufficient to account for the inhibition of the insulin-stimulated $\mathrm{PKB}$ pathway in $\mathrm{C} 2 \mathrm{C} 12$ skeletal muscle cells pretreated with palmitate. J Biol Chem 274:24202-24210

46. Kahn BB, Alquier T, Carling D, Hardie DG (2005) AMPactivated protein kinase: Ancient energy gauge provides clues to modern understanding of metabolism. Cell Metab 1:15-25

47. Barnes BR, Marklund S, Steiler TL et al (2004) The AMPKgamma 3 isoform has a key role for carbohydrate and lipid metabolism in glycolytic skeletal muscle. J Biol Chem 279:38441-38447

48. Bergeron R, Previs SF, Cline GW et al (2001) Effect of 5aminoimidazole-4-carboxamide-1-beta-D-ribofuranoside infusion on in vivo glucose and lipid metabolism in lean and obese Zucker rats. Diabetes 50:1076-1082

49. Iglesias MA, Ye JM, Frangioudakis G et al (2002) AICAR administration causes an apparent enhancement of muscle and liver insulin action in insulin-resistant high-fat-fed rats. Diabetes 51:2886-2894

50. Jorgensen SB, Wojtaszewski JF, Viollet B et al (2005) Effects of alpha-AMPK knockout on exercise-induced gene activation in mouse skeletal muscle. FASEB J 19:1146-1148 